\title{
Hypophosphatasia screening in pediatric age based on records
}

\begin{abstract}
Introduction: hypophosphatasia (HPP) is a congenital disease, characterized by a defect in bone and dentary mineralization, secondary to a deficiency in the biosynthesis of nonspecific tissue isoenzyme of alkaline phosphatase (TNSALP), resulting in decreased levels of alkaline phosphatase (ALP) activity and the extracellular accumulation of its substrates. Our goal was to establish the interest of screening the hospital databases of pediatric patients with low levels of ALP for the diagnosis of HPP.
\end{abstract}

Materials and methods: during the period from September 2016 to September 2017, 23231 patients were tested for ALP, 1752 of them showed low levels of ALP. Based on the clinic, basal disease and that in previous analyses they did not present ALT norm values, 14 cases were selected: 8 cases associated with scoliosis, 1 case associated with early puberty, 1 case of low size, 1 case of liver disease and myasthenia and 1 case of teething problems. $A L P L$ gene was studied in those cases.

Results: the latter case was of an 8-year-old male with a pathological variant of HPP: c.343 348dupACCGCC (p.Thr115 Ala116dup) in exon 5 in dominant heterozygous, inherited from his mother. At the substrate level the levels of pyridoxal-5-phosphate were above $50 \mu \mathrm{g} / \mathrm{L}$

Conclusion: the diagnostic strategy with a high index of clinical suspicion of HPP should include the observation of low levels of serum ALP activity. Our study shows that HPP is an underdiagnosed disease.

An appropriate protocol to detect HPP in a clinical setting in tertiary care hospitals is required.

Keywords: hypophosphatasia, population screening, pediatrics
Volume 9 Issue I - 202 I

\author{
Ignacio Díez-Lopez,' Luis Aldamiz- \\ Echevarría, ${ }^{2}$ Fernando Andrade,' Pablo \\ Suarez,' Domingo González-Lamuño, ${ }^{3}$ \\ María Unceta,' Arantza Arza,' María del Mar \\ Rovira, ${ }^{4}$ Olatz Villate' \\ 'Unidad de Endocrinología Pediátrica, OSI-Araba. Hospital \\ Universitario Araba, Universidad del País Vasco,Vitoria, Álava, \\ Spain \\ ${ }^{2}$ Unidad de Enfermedades Metabólicas Hereditarias. Hospital \\ Universitario de Cruces, Unidad de Endocrinología Pediátrica, \\ OSI-Araba, Hospital Universitario Araba, Universidad del País \\ Vasco, Spain \\ ${ }^{3}$ Servicio de Pediatría, Hospital Universitario Marqués de \\ Valdecilla, Universidad de Cantabria, Santander, Spain \\ ${ }^{4}$ Servicio de Pediatría, Hospital Universitari Germans Trias i \\ Pujol, Universitat Autònoma de Barcelona, Spain
}

\section{Correspondence: Dr. Olatz Villate. Unit of Metabolism,} Cruces University Hospital, Biocruces, Bizkaia Health Research Institute, Plaza de Cruces s/n, 48903, Barakaldo, Spain, Tel (+34) 946006327, Email olatz.villatebejaran@osakidetza.eus

Received: January 27, 2021 | Published: Febrauary I5, 202 I
Abbreviations: HPP, hypophosphatasia; ALP, alkaline phosphatase; TNSALP, tissue-non- specific" isoenzyme alkaline phosphatase; PPi, inorganic pyrophosphate; PEA, phosphoethanolamine; PL, pyridoxal; CEIC, Basque Committee for Clinical Research

\section{Introduction}

Hypophosphatasia (HPP), described in 1948 by Rathbun, ${ }^{1}$ is a congenital disease caused by mutations in the $A L P L$ gene located on chromosome 1p36.12 (MIM171760) ${ }^{2}$ which codifies the "tissuenon- specific" isoenzyme alkaline phosphatase (TNSALP). Mutations cause a diminished ALP's activity and the extracellular accumulation of its substrates. TNSALP is a "cell-surface homodimeric phosphohydrolase" mainly expressed in the bone, liver, kidney and teeth. $^{3}$

HPP can be clinically subdivided into seven groups: perinatal (MIM 241500), infantile (MIM 241500), childhood (MIM 241510), adult (MIM 146300), odontohypophosphatasia (OHPP, MIM 146300), pseudo- HPP and benign prenatal HPP according to the age of onset. ${ }^{4} \mathrm{~A}$ lack of enzymatic activity may be detected in some patients while residual enzyme activity may be observed in others. ${ }^{5}$ Clinical manifestations experienced by individual patients can change over time. ${ }^{6}$

The severe form of this disorder could be explained by autosomal recessive inheritance or by a dominant negative effect on an autosomal dominant mutation, where the gene product from one allele interferes with the monomer-monomer interaction. Mild phenotypes usually present an autosomal dominant mutation. ${ }^{7}$ In Europe, HPP severe forms have an estimated prevalence of $0.6-1 / 300000$ births while the mild forms, with a dominant inheritance, have an estimated prevalence of $1 / 6370$ and in adult clinic population $1 / 1545 .^{8}$

In some populations, prevalence is higher due to a founding effect, as for example the autosomal recessive severe forms of Mennonite population of Manitoba (Canada), where approximately 1 of each 25 people is a carrier of a mutation in the $A L P L$ gene and approximately 1 of each 2500 newborns present a lethal HPP., ${ }^{9,10}$

$A L P L$ gene presents a vast allelic heterogenicity and more than 190 mutations have been described which can explain the different levels of the enzymatic activity, conditioning the wide variability in the clinical expression of the disease. The variability in the inheritance, with recessive and dominant autosomal patterns, and with variable penetrance, makes genetic counselling complicated. ${ }^{11}$

The active isoform of TNSALP is a homodimer and its specific function is to cleave several phosphocompounds such as: inorganic pyrophosphate (PPi), pyridoxal-5-phosphate (PLP), and urine phosphoethanolamine (PEA). ${ }^{12,13}$ Thus, deficiency in TNSALP leads to the extracellular accumulation of these substrates, which results in defects in bone and teeth mineralization, among other clinical features. 
Accumulated extracellular PPi results in impaired mineralization of hard tissue, thus accounting for the cardinal clinical manifestations of HPP, with include loss mineralization, osteomalacia, metatarsal stress fractures, femoral pseudofractures, musculoskeletal, joint pain and premature tooth loss

Deficiency in TNSALP causes a decrease in the dephosphorylation of PLP to pyridoxal (PL), a compulsory step allowing pyridoxine to be transported across the blood-brain barrier. Increases in PLP in the systemic circulation indicates impaired transport of vitamin B6 to the brain, which leads to manifestation of seizures in the severe form of HPP. ${ }^{14}$ The role of phosphoethanolamine (PEA) in the pathophysiology of HPP is yet to be fully understood.

The most difficult phase of diagnosis is the initial orientation since symptoms are adverse, of vary variable severity, of low specificity and, poorly recognized. Once the diagnosis has been suspected, it is confirmed through a biochemical investigation by low levels of serum ALP, in conjunction with the accumulation of natural substrates from TNSALP and clinical and radiographic finding, and later on through a molecular analysis. This stage may necessitate ruling out other causes of low ALP.

Serum ALP is a component of most automated clinical chemistry panels. As such, serum ALP values frequently encountered in the course of medical practice. Low levels of ALP may take place in a sporadic, precipitous way or be persistent, fact that can point us towards its etiology. ${ }^{15}$

The diagnosis of HPP in clinical practice is a challenge. Its low prevalence makes its identification difficult, especially when there is overlapping of symptoms with other more prevalent diseases. In addition, HPP is in general misunderstood and therefore rarely investigated. ${ }^{16}$ In this context, the aim of this study was to assess the low serum ALP levels, excluding cases with known etiologies that cause the decreased values of ALP, in order to assess the potential interest of conducting a selective search in these pediatric patients in centralized biochemical databases.

\section{Materials and methods}

Study subjects: pediatric patients $(<18$ years of age) with ALP values lower than the lower range due to age and sex. Exclusion criteria: HPP family history, absence of another cause of low ALP such as hypomagnesaemia, malnutrition, heart failure, hypothyroidism, celiac disease, chemotherapy, multiorgan failure and lack of signature of informed consent.

Design: a retrospective multicenter observational study has been conducted over a 12-month period (September 2016 to September 2017) without pharmacological intervention. The collaborative hospitals were: Cruces University Hospital (Barakaldo, Spain), Araba University Hospital (Vitoria, Spain) and Marqués de Valdecilla University Hospital (Santander).

Procedure: cases of pediatric patients with low levels of ALP were provided by the centralized biochemistry laboratory, and were identified by the number of history.

The following cut-off points have been taken for the consideration of low ALP levels differentiated by age and $\operatorname{sex}^{17}$ :

Women (age): 0-14 days: <90U/L, 15 days-1 year $<134 \mathrm{U} / \mathrm{L}$, $1-10$ years $<156 \mathrm{U} / \mathrm{L}, 10-13$ years $<141 \mathrm{U} / \mathrm{L}, 13-15$ years $<62 \mathrm{U} / \mathrm{L}$, $15-17$ years $<54 \mathrm{U} / \mathrm{L}, 17-19$ years $<48 \mathrm{U} / \mathrm{L}$.
Men (age): 0-14 days: <90U/L, 15 days-1year: <134U/L, 1-10years: <156U/L, 10-13years: <141U/L, 13-15years: <127U/L, 15-17years: $<89$ U/L, 17-19years $<59$ U/L.

Sample size ${ }^{17}$ : population size: 23231 ; estimated percentage: 0.0005\%; confidence level: $95 \%$; design effect: 1.0 ; precision: $5 \%$; expected sample size to be assessed: 50 .

Among the studied cases, those patients who presented in other analytical studies normal values of ALP have been eliminated, however, cases have been included when there is only a single analysis with low levels of ALP.

After selecting the possible patients, their pediatrician or primary care physician were contacted to review the history and jointly assess whether the patient has a compatible phenotype and meets the selection criteria.

The following parameters were assessed: reason of request for analysis, age, sex, height and weight of the patient, clinical history: data on mobility and walking, appearance of dental manifestations, X-ray tests or appearance of bone manifestations related to hypophosphatasia such as rickets, osteomalacia, bone mineralization alterations or impaired phosphate and calcium metabolism, frequent fractures, impaired mobility, history of seizures dependents of vitamin B6, respiratory commitment, rib/thoracic box deformity and/or psychiatric disorders.

Those patients who met any exclusion criteria were excluded from the study. Selected patients were proposed to proceed with the study and after signing the informed consent, PLP, PEA and genetic studies were performed.

The study has been carried out in accordance with the ethical requirements of the Helsinki declarations, the ICH 'International Conference of Harmonization' Guidelines for Good Epidemiological Practice and the existing regulations in Spain in the Circular 4/2000 of DGFPS (Pharmacovigilance). Respect for the quality of biomedical research projects and respect for the dignity of people during their attainment have been guaranteed, in compliance with Law 41/2002 of 14 November, basic regulatory of patient autonomy and rights and obligations in the field of information and clinical documentation, and Law 14/2007, of July 3, on Biomedical Research. The study has been approved by the Basque Committee for Clinical Research (CEIC) of the Basque Country.

\section{Results}

Patients who were assessed for ALP levels in that period were $23,231,1752$ of them had low levels $(7.5 \%)$. Most of these cases had normal levels of ALP in previous analytical studies, decreasing the number of patients to 67; other cases were excluded as cancer patients, anorexia nervosa, neuromuscular disease, obesity, complex cardiology without clinical or radiological associated phenotype. Finally, a total of 14 cases with low levels of ALP were reported and they were followed up in external metabolopathy consultations. As for the clinic, 8 had scoliosis, 1 case associated with early puberty, 1 case of low height, 1 case of liver disease and myasthenia and 1 case of teething problems. In an expanded analytical study, only one case had elevated levels of pyridoxal-5-phosphate, the genetic study of which confirmed the diagnosis.

\section{Case report}

We present an 8-year-old patient with low ALP levels (71U/L; 
normal range 91-312U/L). Phenotypically asthenia and loss of early teething are the main characteristics.

Non-consanguineous parents, no history of bone or dental pathology, mother with episodes of anxiety were recorded. As a personal background they refer to controlled and normoevolutive pregnancy, with eutocaric delivery at 41 weeks, with no neonatal incidents. Breast-feeding was done for 9 months with introduction of complementary feeding without incidence and normal psychomotor development. He has a correct physical growth with nutritional study and normal physical examination at the time of the visit.

A completed analytical study was conducted and he showed levels of pyridoxal-5-phosphate $>50 \mu \mathrm{g} / \mathrm{L}$ (normal value 5.7-43), with normal urine phosphoethanolamine values of $24 \mathrm{mmol} / \mathrm{mol}$ creatinine.

Suspecting hypophosphatasia, a genetic study was conducted aimed at the study of the $A L P L$ gene by Sanger sequencing detecting the presence of a variant: c.343_348dupACCGCC, aminoacid change: p.Thr115_Ala116dup in exon 5 in heterozygosis, which can be considered pathogenic according to the currently available data confirming the diagnosis. The family study confirmed this variant in the mother showing ALP 24U/L (normal value 30-106), pyridoxal-5phosphat $51.21 \mu \mathrm{g} / \mathrm{L}$ and phosphoethanolamine values in normal urine of $9 \mathrm{mmol} / \mathrm{mol}$ creatinine.

\section{Discussion}

HPP is often overlooked in clinical practice because low serum ALP does not attract the attention of clinicians, limiting the HPP diagnosis. This study, based on the low levels of ALP evidenced in the analyses performed on a paediatric population without any additional clinical features, has been made possible by the existence of an electronic record of them and centralization in a few tertiary hospitals.

Our work has helped to identify an HPP affected patient, and the family study has allowed us to diagnose an adult patient with an unusual clinical phenotype with a dominant heritage. A similar study has recently been published in which no cases were found in the pediatric cohort, but they did not assess cases where only one ALP result was found and it was a single tertiary center. ${ }^{18}$

In the past, $A L P L$ sequencing studies were mostly limited to patients with typical signs of HPP, and a pathogenic mutation could be found in most such individuals. ${ }^{19}$ A study in the United States found

ALPL mutations in 42 of 50 individuals (84\%) with ALP below $30 \mathrm{U} / \mathrm{L},{ }^{20}$ whereas investigators Spain detected $A L P L$ mutations in 36 of $83(43 \%)$ individuals with low ALP. ${ }^{21}$ Selective screening has also been performed in adult patients with persistently low ALP activity who are attended in a rheumatology consultation, where heterozygous or likely pathogenic variants in $A L P L$ were found in $58 \%$ of these individuals ${ }^{22}$ showing that recruited participants on the basis of persistently low ALP levels rather than typical clinical signs of HPP indicate that $A L P L$ mutations are often associated with non-specific symptoms.

It is important to remark that infantile and perinatal HPP cases were detected due to a familiar history of HPP or severe skeletal defects during prenatal and/or perinatal stages. In a recent literature review of cases, the first symptoms of HPP in patients over two years of age are usually close to 4 years. The HPP manifestations in childhood initially experienced dental symptoms, while manifestations among patients in adolescence and adulthood were often predominantly skeletal (low-trauma fractures) and bone pain. ${ }^{23}$ In the adult HPP, a significant proportion also manifested with premature loss of teeth $(35 \%)$, a specific feature for HPP. The principal characteristics of childhood and adult HPP referrals were low ALP with mild bone manifestations. Additionally, a higher frequency of anxiety and depression has been described in adult patients with HPP. The cause of these psychological disorders has not been defined, but TNSALP has been associated with the synthesis of neurotransmitters such as serotonin and dopamine, through its effect on the metabolism of vitamin B6.

Our pediatric patient had dental symptoms, but for his mother the most striking manifestation was psychologically, which supports the idea that a patient with HPP in its evolution may change its phenotype, and the classification established in the HPP is a continuum rather than a fixed clinical phenotype.

A majority of adults with persistently low serum ALP values carry a pathogenic or likely pathogenic variant in the $A L P L$ gene and also have elevated alkaline phosphatase substrate values in serum (PLP) and urine (PEA).

Subjects harboring positive ALPL variants showed lower ALP and higher PLP and PEA values compared with subjects harboring only negative ALPL variants. Approximately half of all subjects harboring positive ALPL variants or ALPL VUS showed elevations in plasma PLP, and three quarters showed elevations in urine PEA.

The active isoform of TNSALP is a homodimer and its specific function is to cleave several phosphocompounds such as: PPi, PLP, and urine PEA. ${ }^{12,13}$ Thus, deficiency in TNSALP leads to the extracellular accumulation of these substrates, which results in defects in bone and teeth mineralization, among other clinical features.

Accumulated extracellular PPi results in impaired mineralization of hard tissue, thus accounting for the cardinal clinical manifestations of HPP, with include loss mineralization, osteomalacia, metatarsal stress fractures, femoral pseudofractures, musculoskeletal, joint pain and premature tooth loss.

Deficiency in TNSALP causes a decrease in the dephosphorylation of PLP to pyridoxal, a compulsory step allowing pyridoxine to be transported across the blood-brain barrier. Increase in PLP in the systemic circulation indicates impaired transport of vitamin B6 to the brain, which leads to manifestation of seizures in the severe form of HPP. ${ }^{14}$ The role of PEA in the pathophysiology of HPP is yet to be fully understood.

Presumed heterozygous carriers for HPP have been known to have low or low-normal serum ALP and mild elevations of ALP substrates, particularly PLP, some studies have found that urine PEA was more frequently elevated and elevated to a greater degree than plasma PLP, we have to take into account that ALP substrates are not always elevated in all patients with HPP. There is no correlation between serum ALP levels and presence of fractures. They have found an increase of serum PLP levels close to significance in patients with fractures.

On the other hand, we have to have in mind patients with HPP frequently (around 50\%) have high serum phosphate values, due to an increase in tubular phosphate reabsorption, contrary to what has been observed in other forms of rickets or osteomalacia.

In the adult period, HPP is most caused by heterozygous $A L P L$ mutations that have a dominant negative effect leading to low serum 
ALP levels. However, only a minority of heterozygous carriers of such dominant mutations seems to develop typical signs of HPP, suggesting low penetrance. ${ }^{19}$

HPP treatment is interdisciplinary and medical, nutritional and in some cases surgical interventions are performed. In the nutritional aspect, it is recommended to decrease calcium intake and a good hydration in the case of hypercalcemia. In case of having normal levels of calcemia and phosphorus or slightly increased, a normocalcemic diet is followed. As a medical treatment, enzymatic replacement therapy is already available (Asfotasa Alfa) as a therapeutic measure, approved in Japan, Canada, the European Union and the USA to treat HPP at pediatric ages. Surgical treatment is reserved for skeletal deformities such as scoliosis or craniosynostosis. Isoled cases of HPP treated with teriparatide have been described with decreased pain and fractures and an improvement in biochemical and densitometric parameters, indicating that the response may vary depending on the mutation of TNSALP. ${ }^{24,25}$

Due to the characteristic hypomineralization of this disease, HPP is often misdiagnosed as osteoporosis and treated with antisorptive drugs, such as bisphosphonates. These drugs worsen the prognosis of HPP.

These show the importance of accurate diagnosis and evaluation of possible HPP in patients in whom a treatment with bisphosphonates is considered. ${ }^{26}$

This study allowed us to diagnose a case of a pedriatic patient and it was the index case for the familiar study. We have diagnosed his mother, which makes this methodology promising for the search for cases with HPP. Currently the centralization of analytical determinations, their automation and registration favor their implementation, and we must also keep in mind that automation allows establishing procedural algorithms in such a way that an automatic mode in the case of a low ALP result, analytical determinations such as PLP can be required.

Among the biases of this work, selection on the basis that those who were believed to have persistent low levels of ALP have a clear cause have not been studied, and cases without clinics can therefore go undiagnosed. We have to keep in mind that in normal situations of scoliosis there are high levels of ALP.

Undoubtedly, more studies are needed to assess the use of these databases, along with specific algorithms, to diagnose rare diseases such as HPP.

\section{Conclusion}

The development of specific algorithms allows a better approach to the diagnostic challenge posed by HPP, especially whose phenotypic overlap with other forms of bone fragility can lead to inadequate treatments that worsen the prognosis. The diagnostic strategy with a high index of clinical suspicion of HPP should include the observation of low levels of serum ALP activity, radiological abnormalities and the accumulation of substrates of ALP.

Our study shows that HPP is an underdiagnosed disease. An appropriate protocol to detect HPP in a clinical setting in tertiary care hospitals is required.

\section{Acknowledgments}

Authors would like to thank patients for their collaboration in our study.

\section{Statement of Authorship}

LAE, ID, FA PS, DGL, MU, AA, MMR and OV collected data and reviewed the manuscript. MMR contributed to manuscript writing. LAE, FA and OV designed the study, drafted the initial manuscript and reviewed and revised the manuscript. All authors approved the final manuscript.

\section{Funding}

None.

\section{Conflicts of interest}

The authors declare no conflict of interest.

This research received funding from Alexion Pharmaceuticals, Inc to conduct the study.

\section{References}

1. Rathbun JC. Hypophosphatasia, a new developmental anomaly. Am J Dis Child. 1948;75:822-831.

2. Weiss MJRK, Henthorn PS, Lamb B, et al. Structure of the human liver/ bone/kidney alkaline phosphatase gene. J Biol Chem. 1988;263:1200212010

3. Orimo $\mathrm{H}$. The mechanism of mineralization and the role of alkaline phosphatase in health and disease. J Nippon Med Sch. 2010;77:4-12.

4. Rockman-Greenberg C. Hypophosphatasia. Pediatr Endocrinol Rev. 2013;10:380-388.

5. Whyte MP, Zhang F, Wenkert D, et al. Hypophosphatasia: validation and expansion of the clinical nosology for children from 25 years experience with 173 pediatric patients. Bone. 2015;75:229-239.

6. Mori M,DeArmey SL, WeberTJ, etal.Case series: Odontohypophosphatasia or missed diagnosis of childhood/adult-onset hypophosphatasia? - Call for a long-term follow-up of premature loss of primary teeth. Bone Rep. 2016:5:228-232.

7. Whyte MP. Hypophosphatasia-aetiology, nosology, pathogenesis, diagnosis and treatment. Nat Rev Endocrinol. 2016;12:233-246.

8. McKiernan FE, Berg RL, Fuehrer J. Clinical and radiographic findings in adults with persistent hypophosphatasemia. $J$ Bone Miner Res. 2014;29:1651-1660

9. Mornet E, Yvard A, Taillandier A, et al. A molecular-based estimation of the prevalence of hypophosphatasia in the European population. Ann Hum Genet. 2011;75:439-445.

10. Leung EC, Mhanni AA, Reed M, et al. Outcome of perinatal hypophosphatasia in manitoba mennonites: A retrospective cohort analysis. JIMD Rep. 2013;11:73-78.

11. Mornet E, Beck C, Bloch-Zupan A, et al. Clinical utility gene card for: hypophosphatasia. Eur J Hum Genet. 2011;19.

12. Russell RG. Excretion of inorganic pyrophosphate in hypophosphatasia Lancet. 1965;2:461-464.

13. Macfarlane JD, Poorthuis BJ, Mulivor RA, et al. Raised urinary excretion of inorganic pyrophosphate in asymptomatic members of a hypophosphatasia kindred. Clin Chim Acta. 1991;202:141-148.

14. Mornet E. Hypophosphatasia. Best Pract Res Clin Rheumatol. 2008;22(1):113-127.

15. McKiernan FE, Shrestha LK, Berg RL, et al. Acute hypophosphatasemia. Osteoporos Int. 2014;25(2):519-523. 
16. Maman E, Borderie D, Roux C, et al. Absence of recognition of low alkaline phosphatase level in a tertiary care hospital. Osteoporos Int 2016;27(3):1251-1254.

17. Colantonio DA, Lianna $\mathrm{K}$, Man KC, et al. Closing the gaps in pediatric laboratory reference intervals: A CALIPER database of 40 biochemical markers in a healthy and multiethnic population of children. Clinical Chemistry. 2012:58;854-868.

18. García-Fontana C, Villa-Suárez JM, Andújar-Vera F, et al. Epidemiological, clinical and genetic study of hypophosphatasia in a Spanish population: identification of two novel mutations in The Alpl gene. Sci Rep. 2019;9:9569.

19. Fauvert D, Brun-Heath I, Lia-Baldini AS, et al. Mild forms of hypophosphatasia mostly result from dominant negative effect of severe alleles or from compound heterozygosity for severe and moderate alleles. BMC Med Genet. 2009;10:51.

20. McKiernan FE, Dong J, Berg RL, et al. Mutational and biochemical findings in adults with persistent hypophosphatasemia. Osteoporos Int 2017;28:2343-2348
21. Tenorio J, Álvarez I, Riancho-Zarrabeitia L, et al. Molecular and clinical analysis of ALPL in a cohort of patients with suspicion of Hypophosphatasia. Am J Med Genet A. 2017;173:601-610.

22. Rauch F, Bardai G, Rockman-Greenberg C. ALPL mutations in adults with rheumatologic disorders and low serum alkaline phosphatase activity. $J$ Bone Miner Metab. 2019.

23. Szabo SM, Tomazos IC, Petryk A, et al. Frequency and age at occurrence of clinical manifestations of disease in patients with hypophosphatasia: a systematic literature review. Orphanet J Rare Dis. 2019;14:85.

24. Camacho PM, Painter S, Kadanoff R. Treatment of adult hypophosphatasia with teriparatide. Endocr Pract. 2008;14:204-208.

25. Laroche M. Failure of teriparatide in treatment of bone complications of adult hypophosphatasia. Calcif Tissue Int. 2012;90:250.

26. Sutton RA, Mumm S, Coburn SP, et al. "Atypical femoral fractures" during bisphosphonate exposure in adult hypophosphatasia. J Bone Miner Res. 2012;27:987-994 